ORIGINAL ARTICLE

\title{
Imaging infants with head injury: effect of a change in policy
}

\author{
J G Browning, M J Reed, A G Wilkinson, T Beattie
}

Emerg Med J 2005;22:33-36. doi: 10.1136/emj.2004.016196

See end of article for authors' affiliations ......................

Correspondence to:

Miss J G Browning,

Accident and Emergency Department, Royal

Hospital for Sick Children,

Sciennes Road, Edinburgh EH7 5HD, UK;

jenbrowning@hotmail.com

Accepted for publication 13 July 2004
Objective: Head injury is one of the commoner injuries presenting to the emergency department (ED). Infants are hard to assess clinically and emphasis has been placed on radiological examination. Skull radiographs, however, are not a reliable indicator of intracranial injury. As a result of this the policy in this ED was revised so that skull radiographs were only to be performed in those infants less than 1 year with visible evidence of head injury or a suspicious history for non-accidental injury.

Methods: Retrospective cohort study of all infants less than 1 year who presented with head trauma to the ED of a paediatric teaching hospital between 1 August 1998 and 31 July 1999, and between 1 August 2002 and 31 July 2003. Hospital notes and radiology computer systems were examined and data were collected and analysed.

Results: 181 infants aged less than 1 year presenting to the ED in 1998/9 and 190 infants in 2002/3. Altogether $140(77.3 \%)$ infants had a skull radiograph in 1998/9, five $(3.6 \%)$ identified skull fractures. During 2002/3, $56(29.5 \%)$ infants had a radiograph, a reduction of $47.5 \%$, of which three $(5.4 \%)$ had skull fractures. All fractures had reported haematomas to their scalp. The change in policy decreased the total radiation dose to the population by $9.4 \mathrm{mSv}$. No significant injuries were missed as a result of the change in policy.

Interpretation: In infants under 1 year, unless non-accidental injury is suspected, it is suggested that skull radiographs should only be performed when there are visible signs of a head injury.
$\mathrm{H}$ ead injury (HI) is one of the commoner injuries presenting to the emergency department (ED). A substantial number of these are found in children. Infants differ from older children and adults ${ }^{1}$ in that clinical assessment is more difficult and the risk of non-accidental injury (NAI) is high.

While clinical assessment and judgement are important, emphasis has, in the past, been placed on radiological investigation as an adjunct to effective and safe treatment. ${ }^{1-3}$ However, the use of radiographs is not without difficulty. The radiation dose is significant and many junior doctors find skull radiographs difficult to interpret in infants with fractures sometimes missed by non-radiological staff. ${ }^{4}$ False positives also occur because of sutures and vascular markings being mistaken for fractures.

The presence of a skull fracture is not a reliable indicator of intracranial injury in infants as significant intracranial injury occurs more commonly in the absence of a skull fracture than in adults. ${ }^{56}$ Computed tomography (CT) is the gold standard for detection of intracranial injury. However, this often requires sedation with all of its attendant difficulties. ${ }^{7}$ Ultrasound (US) is another imaging technique that can be used in infants in whom the fontanelles are still open. It can only reliably exclude subdural and intraventricular haemorrhage, and may miss subtler intracranial abnormality. ${ }^{8}$

National Institute for Clinical Effectiveness (NICE) guidelines suggest skull radiographs have a role combined with high quality inpatient observation where CT unavailable. They also suggest that skull radiographs have a role in the detection of NAI. ${ }^{9}$ The Scottish Intercollegiate Guideline Network (SIGN) guidelines place more emphasis on skull radiographs where there are risk factors for fracture or intracranial injury, although they acknowledge that skull fractures in children are less commonly associated with intracranial injury, and therefore their detection is less helpful than in the adult population. ${ }^{10}$

In 1997 Lloyd et al ${ }^{4}$ published a paper in which they concluded that skull radiographs are not a reliable predictor of intracranial injury. In the light of these findings we critically evaluated our own practice. As a result we revised the policy in our ED so that skull radiographs were only to be performed in children less than 1 year with visible evidence of HI a suspicious history for NAI (box). This new policy is compatible with the recommendations issued by NICE although some important differences exist.

The purpose of this paper is to evaluate the effect of this change in policy, in particular with regard to the recent management guidelines published by NICE. There are scarce published data on HI specific to this age group and this evidence may help the development of similar policies and future guidelines.

\section{METHOD}

This study is a retrospective cohort study of all infants less than 1 year who presented with head trauma to the ED of a paediatric teaching hospital between 1 August 1998 and 31 July 1999 (period 1), and between l August 2002 and 31 July 2003 (period 2). The first period was chosen as it represents the last full year before the revision of the departmental HI policy, and the second period as it represents the most recent full year since the introduction of the revised HI policy (box).

Subjects were identified by a search of the ED database looking for all patients less than 1 year with a presenting or discharge diagnosis of HI. The radiology department database was also searched to identify all those having a skull radiograph, USS of the head, or CT of the head during this period. Hospital records were then retrieved and details of their injury, treatment, imaging, and subsequent clinical course were collected on a specifically designed form. Records were searched to identify infants who returned, deteriorated, or developed complications after initial discharge. As our

Abbreviations: $\mathrm{NAl}$, non-accidental injury; $\mathrm{HI}$, head injury; $\mathrm{ED}$, emergency department; CT, computed tomography; US, ultrasonography 
Present guidelines for radiological imaging in head injuries (RHSCE emergency department's head injury policy)

\section{Skull radiograph}

- Age $<1$ year and visible evidence of head injury or suspicious history for NAl

\section{Admission for observation and/or CT}

- Loss of consciousness more than five minutes

- Amnesia

- Persisting headache, vomiting, or lethargy

- Coagulation or bleeding diathesis (also needs discussion with haematology)

\section{Immediate CT head}

- GCS less than 14

- Focal neurological signs

- Seizure (focal or prolonged)

- Signs of base of skull fracture

- ? Penetrating injury

- ? Depressed fracture

hospital is the only admitting paediatric hospital in the area, all infants in this category would have re-attended here, or would have been admitted here.

Skull radiographs were interpreted initially by the ED or surgical staff on duty, and were then reported by the on call consultant paediatric radiologist the following day, whose report was taken as the gold standard. Any patient who had a skull fracture and was missed by non-radiological staff was recalled for review.

Our inclusion criteria were: age less than 1 year at time of presentation, diagnosis of HI. Exclusion criteria were: age l year or greater at time of presentation, infants having imaging for reasons other than trauma.

Completed forms were collected for data entry and analysis using Microsoft Excel (Microsoft, Seattle). Fisher's exact test was used to compare categorical data, a binomial test to compare the number of radiographs in each period and the Mann-Whitney $U$ test to compare non-parametric distributions of continuous variables.

\section{RESULTS}

Altogether 371 infants were identified as fitting the inclusion criteria, 181 in period 1 (1998/9) and 190 in period 2 (2002/3). Table 1 shows the results.

We were unable to review two sets of missing notes, one from each period, but we were able to glean sufficient data to include both patients in the study. Both patients were discharged home without any imaging and neither represented to the department.
There was no significant difference in infant demographics between periods (table 1). Fifty two per cent were male and the median age was 8 months.

During 1998/99, 140 (77\%) infants had a radiograph of which 135 were normal. Five (3.6\%) identified skull fractures. All of these infants had reported haematomas to their scalp. ED staff missed one parietal fracture. However, this infant was admitted for observation because of drowsiness. During 2002/3, 56 infants had a radiograph, a reduction in skull radiographs of $60.0 \%$. Three $(5.4 \%)$ of these showed a fracture. Again all three had scalp haematomas. Eighty six fewer radiographs were carried out in period 2 reducing the radiation dose to the population by $9.4 \mathrm{mSv}$ (table 2 ).

Table 3 shows the type of injury and whether infants were radiographed or not. In 1998/9, 50 (27.6\%) infants had skull radiographs despite no visible signs of injury compared with four $(2.1 \%)$, in $2002 / 3$. These latter radiographs were done because of mechanism of injury.

Figure 1 shows the mechanisms of injury for the two periods. As expected the commonest mechanism of injury in both periods is a fall less than one metre. Five $(62.5 \%)$ of the fractures occurred from falls less than one metre including the depressed fracture, two $(25 \%)$ from falls greater than one metre, and the multiple skull fractures occurred from falling down stairs. The infant with the subdural haematoma was thought to have been shaken and presented with a reduced conscious level.

In 1998/9, three infants underwent CT (1.7\%). One infant presented with a decreased conscious level, bilateral retinal haemorrhages, and bulging fontanelle despite no visible signs of HI and went directly for CT after resuscitation and stabilisation. This showed a subdural haematoma. Her injuries were considered to be non-accidental. A second infant underwent CT acutely; this confirmed multiple skull fractures with no intracranial injury. A third scan was done on an infant who returned four days after HI with intermittent vomiting, irritability, and drowsiness. It was normal and an intercurrent viral infection was diagnosed. In $2002 / 3$, only one infant had CT $(0.5 \%)$, which confirmed a depressed skull fracture. This meant an overall radiation dose of $21.16 \mathrm{mSv}$ in $1998 / 9$ with a reduction of $63.3 \%$ to $7.76 \mathrm{mSv}$ in $2002 / 3$.

US was used as an alternative to CT in seven patients. All of these infants were admitted and none of the scans were performed acutely. In period 1 , four infants had US scans the following day; two confirmed fractures shown on radiography and two were normal. The normal scans were done because of persistent vomiting and drowsiness. In period 2, three infants had US scans; two were done the following day and confirmed fractures shown on radiography. The other US scan was done six days after HI when the infant returned with a parietal swelling but no other symptoms or signs. The scan confirmed a scalp haematoma, with no other injury, and the infant was discharged. None of the scans showed any intracranial abnormality.

Table 1 Comparison between the two study periods, one before and one after the abolition of skull radiographs

\begin{tabular}{llll}
\hline & Period 1 1998/9 & Period 2 2002/3 & p Value \\
\hline Head injuries < 1 year & 181 & 190 & $0.68^{*}$ \\
Number of male infants & $94(52)$ & $98(52)$ & $1.00 \dagger$ \\
Age (months) median (IQR) & $7.9(4.3-9.7)$ & $7.4(5.0-9.5)$ & $0.96 \ddagger$ \\
Skull fracture on radiograph & $5 / 140(3.6)$ & $3 / 54(5.6)$ & $0.69 \dagger$ \\
Abnormal CT & $2 / 3(67)$ & $1 / 1(100)$ & $0.36 \dagger$ \\
Abnormal US & $2 / 4(50)$ & $2 / 3(67)$ & $1.0 \dagger$ \\
\hline
\end{tabular}

*Binomial test; †Fisher's exact test; ¥Mann-Whitney $U$ test. Percentages are shown in parentheses unless stated otherwise. 
Table 2 Comparison of radiation dose between the two periods

\begin{tabular}{llll}
\hline & Period 1 1998/9 & Period 2 2002/3 & p Value \\
\hline Skull radiograph & 140 & 54 & $<0.001^{*}$ \\
Number (\%) of two views & $111(79)$ & $45(83)$ & $0.69^{*}$ \\
Number (\%) of three views & $29(21)$ & $9(17)$ & \\
Skull radiograph total radiation dose & $15.16 \mathrm{mSv}$ & $5.76 \mathrm{mSv}$ & $<0.001 \dagger$ \\
Computed tomography & 3 & 1 & $0.36^{*}$ \\
Total radiation dose & $21.16 \mathrm{mSv}$ & $7.76 \mathrm{mSv}$ & $<0.001 \dagger$ \\
\hline *Fisher's exact test; †Mann-Whitney U test. & & \\
\hline
\end{tabular}

Twenty five (13.8\%) infants were admitted in period 1, compared with $19(10 \%)$ infants in period 2. Two patients, one in each group, required high dependency care overnight. All other admissions were observed overnight on a surgical ward. All of the infants with fractures were admitted. Eight infants, six in period 1 and two in period 2, were admitted because of an ED staff diagnosed fracture whose subsequent radiological reports were normal. This may account for the higher admission rate in period 1 as these infants were all admitted for their suspected skull fracture.

Other reasons for admission included: persistent vomiting, drowsiness, irritability, mechanism of injury, parental anxiety, and social problems. None of the infants required operative treatment and all survived to hospital discharge.

\section{DISCUSSION}

There are no specific guidelines on the treatment of infants less than 1 year with head injuries, ${ }^{11}$ and there is little published in the literature. Our guidelines were based on research by Lloyd et al, who suggested that too many skull radiographs were being requested with resultant unnecessary exposure to radiation. ${ }^{4}$ After careful review of this and other literature we changed our policy in an attempt to decrease the radiation dose to the infant population.

Our results show that the change in our policy to performing skull radiography in those children less than l year only if there was visible evidence of HI, or a history raising suspicion of NAI decreased the radiation dose significantly $(9.4 \mathrm{mSv})$, with no detriment to any infant in terms of missed injury. We should now consider whether we can decrease the radiation dose further. Clinical features, such as drowsiness, lethargy, etc, are possibly more powerful indicators of clinical course than radiographs. This has been illustrated by the infant with the missed skull fracture correctly admitted because of lethargy. The skull radiograph made no difference to overall management or outcome. Our policy would have also detected most of those with occult intracranial in Greenes' study. ${ }^{12}$

Previous studies have shown that the presence of a scalp haematoma in an infant increases the risk of underlying skull factures. ${ }^{513-17}$ Our data confirm this as all infants with skull fractures had recorded scalp haematomas.
There were 13 infants in period 2 who had reported scalp haematomas but despite this were discharged home without a skull radiograph. The reasons for the breach in protocol included: insignificant injuries, trivial mechanism of injury, and delayed presentation. None of them had any other symptoms or signs on examination and they were all discharged home. One infant re-presented the following day with tonsillitis but there were no other re-presentations suggesting that no serious injuries were missed.

Despite the change in protocol, four children in 2002/3 with no symptoms, and no visible signs of HI had a skull radiograph. The main reason for this was concern regarding the mechanism of injury. None of these had a fracture and all were discharged home. The role of mechanism of injury is unclear. Chambers et al found that falls in excess of 1.5 metres increased the risk of injury four times that of falls from 1.5 metres or less. ${ }^{18}$ Shaken infants show no external signs of HI, although there may be other signs of NAI. Read et $a l^{19}$ concluded that all children with a diminished level of consciousness need to undergo an urgent CT scan. Unless we use other imaging modalities more frequently there is limited scope for decreasing skull radiographs further.

One limitation of our study is that not all patients had a CT scan, the gold standard to detect intracranial injury. However, this would be ethically unacceptable. One delayed CT scan was done because of re-presentation with symptoms attributable to HI. However, none of the other infants deteriorated and there were no other significant representations suggesting there were no other serious intracranial injuries.

Inflicted HI has been recognised as the most common cause of injury related deaths in infants..$^{20}{ }^{21}$ The risk of NAI is important, and seven of the 371 infants presentations were thought to be suspicious. All were investigated further and three of the infants were placed on the child protection register. The infant who had been shaken was correctly identified by clinical signs and went directly for a CT scan. A skull radiograph done subsequently as part of the skeletal survey was normal. Staff in the ED should always be suspicious of NAI in infants presenting with symptoms and signs of $\mathrm{HI}$.

Table 3 Type of injuries

\begin{tabular}{|c|c|c|c|c|}
\hline \multirow[b]{2}{*}{ Type of injury } & \multicolumn{2}{|c|}{ Period 1 1998/9 } & \multicolumn{2}{|c|}{ Period 2 2002/3 } \\
\hline & Radiography & No radiography & Radiography & No radiography \\
\hline Haematoma to face & 57 & 13 & 26 & 48 \\
\hline Laceration to face & 4 & 5 & 0 & 3 \\
\hline Haematoma to scalp & $25^{*}$ & 8 & $23+$ & 13 \\
\hline Laceration to scalp & 1 & 0 & 1 & 2 \\
\hline No visible signs of injury & 50 & $14 \ddagger$ & 4 & 69 \\
\hline Other & 2 & 1 & 0 & 0 \\
\hline Notes missing & 0 & 1 & 0 & 1 \\
\hline
\end{tabular}

*Four parietal fractures, one multiple fractures-boggy. †One depressed parietal fracture-boggy, one occipital fracture, one parietal fracture. †One subdural-NAl, skull radiography done for skeletal survey-normal. 


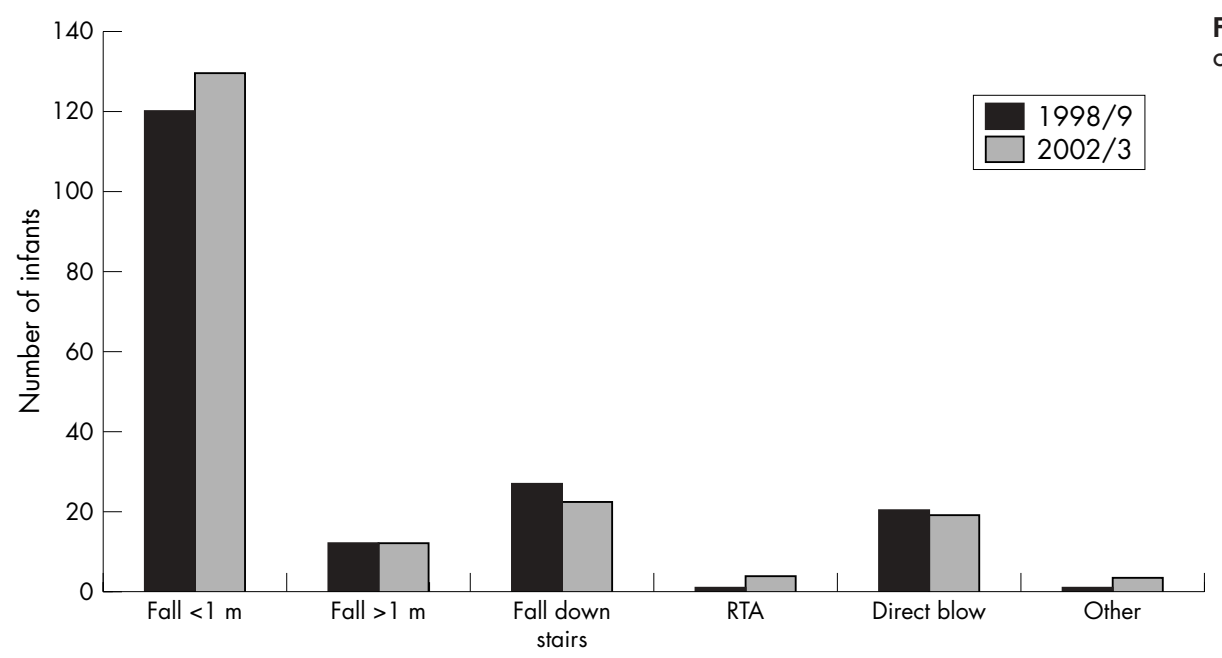

Figure 1 Graph to show mechanism of injury.

In summary, in infants under 1 year, unless NAI is suspected, we suggest that skull radiographs should only be performed when there are visible signs of a HI. Further imaging should be restricted to those infants with changed level of consciousness, or who do not improve with time.

\section{CONTRIBUTORS}

$\mathrm{T}$ Beattie and A Wilkinson conceived the idea of the study and were responsible for the change in protocol. J Browning and M Reed were responsible for study design, data collection, and interpretation. $\mathrm{J}$ Browning wrote the initial draft of the report and all authors participated in its review and finalisation.

\section{ACKNOWLEDGEMENTS}

We thank Rik Smith at the Scottish Trauma Audit Group for statistical advice, and the radiographers in the radiological department at RHSCE for help with retrieval of CT and radiological data.

\section{Authors' affiliations}

J G Browning, M J Reed, T Beattie, Accident and Emergency Department, Royal Hospital for Sick Children, Edinburgh, UK

A G Wilkinson, Radiology Department, Royal Hospital for Sick Children

Funding: none.

Conflicts of interest: none declared.

Ethical approval: not required.

\section{REFERENCES}

1 Leonidas JC, Ting W, Binkiewicz A, et al. Mild head trauma in children: when is a roentgenogram necessary? Pediatrics 1982;69:139-43.

2 Ros SP, Cetta F. Are skull radiographs useful in the evaluation of asymptomatic infants following minor head injury? Pediatr Emerg Care 1992;8:328-30.

3 Pietrzak M, Jagoda A, Brown L. Evaluation of minor head trauma in children younger than two years. Am J Emerg Med 1991;9:153-6.

4 Lloyd DA, Carty H, Patterson M, et al. Predictive value of skull radiography for intracranial injury in children with blunt head injury. Lancet 1997;349:821-4.
5 Shane SA, Fuchs SM. Skull fractures in infants and predictors of associated intracranial injury. Pediatr Emerg Care 1997;13:198-203.

6 Levi L, Guilburd JN, Linn S, et al. The association between skull fracture, intracranial pathology and outcome in pediatric head injury. $\mathrm{Br} J$ Neurosurg 1991:5:617-25.

7 Scottish Intercollegiate Guidelines Network (SIGN). Safe sedation of children undergoing diagnostic and therapeutic procedures. Publication number 58. Edinburgh: SIGN, 2002.

8 Graham CA, O'Toole SJ, Haddock G. Neonatal head injuries. Emerg Med J 2000;17:334-6.

9 National Institute for Clinical Excellence (NICE). Head injury. Triage, assessment, investigation and early management of head injuries in infants, children and adults. Clinical guideline number 4. London: NICE, 2003.

10 Scottish Intercollegiate Guidelines Network (SIGN). Early management of patients with head injury. Publication number 46. Edinburgh: SIGN, 2000.

11 Schutzman SA, Barnes P, Duhaime AC, et al. Evaluation and management of children younger than two years old with apparently minor head trauma: proposed guidelines. Pediatrics 2001;107:983-93.

12 Greenes DS, Schutzman SA. Occult Intracranial Injury in Infants. Ann Emerg Med 1998;32:680-6.

13 Greenes DS, Schutzman SA. Infants with isolated skull fracture: what are their clinical characteristics, and do they require hospitalisation? Ann Emerg Med 1997;30:253-9.

14 Quayle KS, Jaffe DM, Kuppermann N, et al. Diagnostic testing for acute head injury in children: When are head computerised tomography and skull $x$-rays indicated? Pediatrics [Online] 1997;99:E11.

15 Kleinman PK, Spevak MR. Soft tissue swelling and acute skull fractures. J Pediatr 1992;121:737-9.

16 Greenes DS, Schutzman SA. Clinical significance of scalp abnormalities in asymptomatic infants. Pediatr Emerg Care 2001;17:88-92.

17 Greenes DS, Schutzman SA. Clinical indicators of intracranial injury in headinjured infants. Pediatrics 1999;104:861-7.

18 Chalmers DJ, Marshall SW, Langley JD, et al. Height and surfacing as risk factors for injury in falls from playground equipment: a case-control study. Injury Prevention 1996;2:98-104.

19 Read HS, Johnstone AJ, Scobie WG. Skull fractures in children: altered conscious level is the main indication for urgent CT scanning. Injury 1995;26:333-4.

20 Billmire ME, Myres PA. Serious head injury in infants: Accident or abuse? Pediatrics 1985;75:340-2

21 Duhaime AC, Christian CW, Rorke LB, et al. Non-accidental head injury in infants: the "shaken-baby syndrome." N Engl J Med 1998;338:1822-9. 